\title{
Dielectric Barrier Discharge Applicator for Heating Carbon Nanotube-loaded Interfaces and Enhancing 3D-Printed Bond Strength
}

\section{$\underline{\text { Supporting Information }}$}

Charles B. Sweeney, ${ }^{1,2,3}$ Matthew L. Burnette, ${ }^{4}$ Martin J. Pospisil, ${ }^{3}$ Smit A. Shah, ${ }^{3}$ Muhammad Anas, ${ }^{3}$ Blake R. Teipel, ${ }^{1}$ Bryan S. Zahner, ${ }^{1}$ David Staack, ${ }^{4}$ Micah J. Green, ${ }^{2,3 *}$

1. Essentium 3D, 19025 Heatherwilde Blvd., Pflugerville, Texas 78660, United States

2. Department of Materials Science and Engineering, Texas A\&M University, College Station, TX, USA, 77843

3. Artie McFerrin Department of Chemical Engineering, Texas A\&M University, College Station, TX, USA, 77843

4. Department of Mechanical Engineering, Texas A\&M University, College Station, TX, USA, 77843

*corresponding author, micah.green@tamu.edu

\section{Section S1. Carbon Nanotube Loading}

The multi-walled carbon nanotubes used in this study have an average diameter of $\sim 10 \mathrm{~nm}$ and an average length of $\sim 2 \mu \mathrm{m}$ and are commercially compounded into a masterbatch by the supplier.

In our prior work, we determined the following relationship between heating rate and filler loading: At low CNT loadings, the CNT-polymer composite layer heating is relatively low. The heating rate increases markedly once the CNT loading exceeds the percolation threshold. However, at high CNT content, the composite can become conductive enough that decreased penetration depth of the electric field actually causes a decrease in heating rate. This relationship can be written as

$P \sim \int \sigma|E| d V$

where $\mathrm{P}$ is the power dissipated as heat, $\sigma$ is the conductivity, and $\mathrm{E}$ is the electric field. As conductivity increases, the penetration depth of the field decreases. This is the reason for the non-monotonic relationship between CNT loading and observed heating rate. This is true for a wide range of applied electromagnetic field frequencies. ${ }^{1,2}$ 


\section{Section S2. COMSOL Multiphysics Modeling}

The computational model was solved using COMSOL Multiphysics 5.1 (COMSOL Inc. Los Angeles, CA) finite element software. Our model geometry utilized the same dimensions as its experimental counterpart. Using the built-in physics module in COMSOL, the Electromagnetic Wave (Equation 1) equation was solved. The following PDE describes the systems:

$$
\nabla \times \mu_{r}^{-1}(\nabla \times \boldsymbol{E})-k_{0}^{2}\left(e_{r}-\frac{j \sigma}{\omega e_{0}}\right) \boldsymbol{E}=0,
$$

The variables used in Equation 1 are as follows: $\mu_{r}$ is the relative permeability of the material, $\epsilon_{r}$ is the relative permittivity of the material, $k_{0}$ is the known wave number, $\sigma$ is the electric conductivity of the material, and $\omega$ is the operating frequency.

In the Electromagnetic Wave module, our boundary conditions for the 3D printed part consisted of two Perfect Electric Conductors (Equation 2) where the plasma formed and where the printer nozzle contacted the surface and two Lumped Ports (Equation 3) for the gaps between the plasma and the printer nozzle at the top surface. See Figure S1 for schematic.

$$
\begin{gathered}
\boldsymbol{n} \times \boldsymbol{E}=0 \\
Z=\frac{V}{I}
\end{gathered}
$$

Here, $\mathbf{n}$ is the vector normal to the boundary, I is the current produced by the DBD plasma, $\mathrm{Z}$ is the lumped port impedance, and $\mathrm{V}$ is the applied voltage.

The mesh for the model domain was created using a tetrahedral mesh. The total number of degrees of freedom used was 94,774 .

The built-in Frequency-Domain Study solver was used to solve the equation for the electric field distribution. The frequency of the simulation was set to $30 \mathrm{MHz}$. 


\section{COMSOL Parameters:}

3D part geometry

Height $3 \mathrm{~mm}$

Length $8.8 \mathrm{~cm}$

Depth $5 \mathrm{~cm}$

\section{Lumped Port parameters}

Type of lumped port: User defined

Height of lumped port: $3 \mathrm{~mm}$

Width of lumped port: $6 \mathrm{~mm}$

Direction between lumped port terminals:

Left port: [1, 0, 0]; Right port: [-1, 0, 0]

Terminal type: Cable

Wave excitation: On

Voltage: $8.2 \mathrm{kV}$

Port phase: 0

Characteristic impedance: $50 \Omega$

Material properties

Relative permittivity: 5

Density: 1150 [kg/m³]

Relative permeability: 1

Electrical conductivity: 10 [S/m]

Initial values in model

Electric field $0 \mathrm{~V} / \mathrm{m}$ 


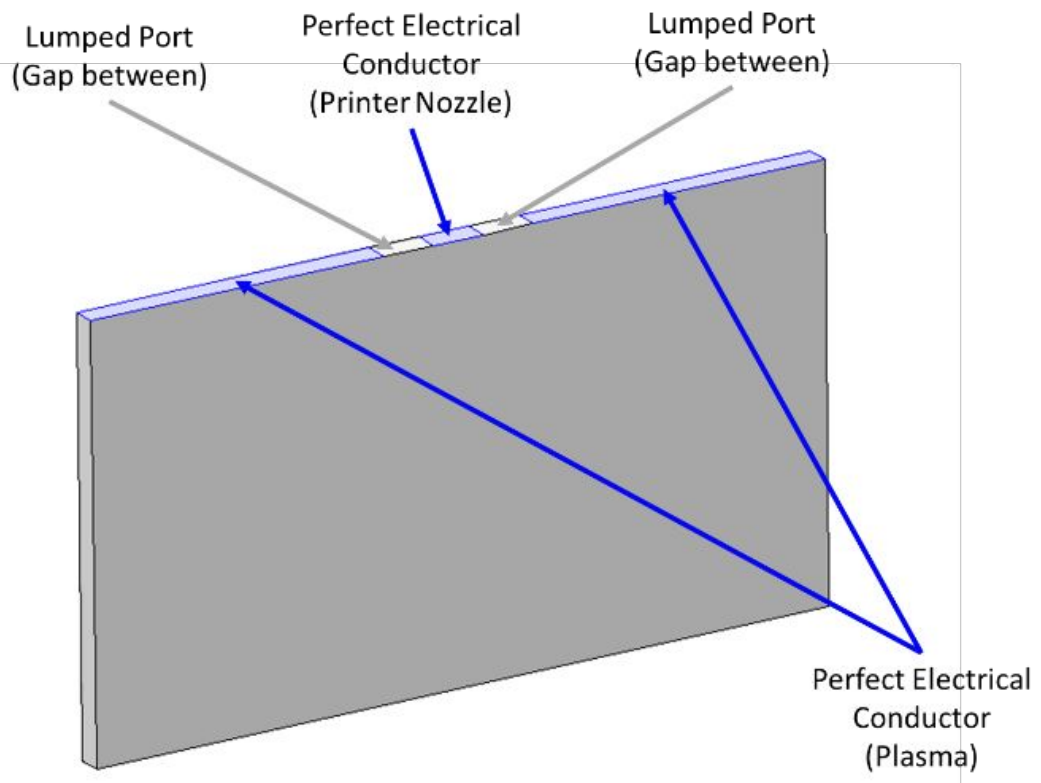

Figure S1: Schematic shows boundary conditions for simulation of 3D-printed part. 


\section{Section S3. Equivalent Circuit}

The mechanism for heating is best understood by analyzing an equivalent circuit model of the system. An AC current source is connected in series to the high voltage plane in the applicator disc. The dielectric barrier layer used in the disc has a characteristic capacitance $\mathrm{C}_{\mathrm{d}}$ associated with it given by the material properties of the dielectric, and the geometry of the disc. In between the disc and the 3D-printed part is an air gap, typically between $0.5-2 \mathrm{~mm}$, where the plasma forms. In the simplest case, DBDs may be modeled with an equivalent circuit (Figure S2) consisting of a resistor $R_{p}$ and capacitor $C_{p}$ in parallel ${ }^{3}$. In reality, the resistor is a transient element that is switching on and off at a rate consistent with the driving frequency of the power source. The resistance of the plasma is based on the composition of the atmosphere and the power associated with the DBD. The resistance of the plasma results in some heating of the air, as well as light emissions from excited gaseous species. The DBD couples directly to the 3D-printed part, which is resistive based on the small amount of CNTs added to the thermoplastic feedstock filament. The composition of the nanocomposite material is selected such that the electric current from the DBD couples efficiently to the printed layers, resulting in significant Joule heating. The current flowing through the 3D-printed part flows to the 3D printer nozzle which is electrically grounded to the $\mathrm{AC}$ current source. Based on this circuit configuration, our technology may be defined as an asymmetric resistive dielectric barrier discharge ${ }^{4,5}$.
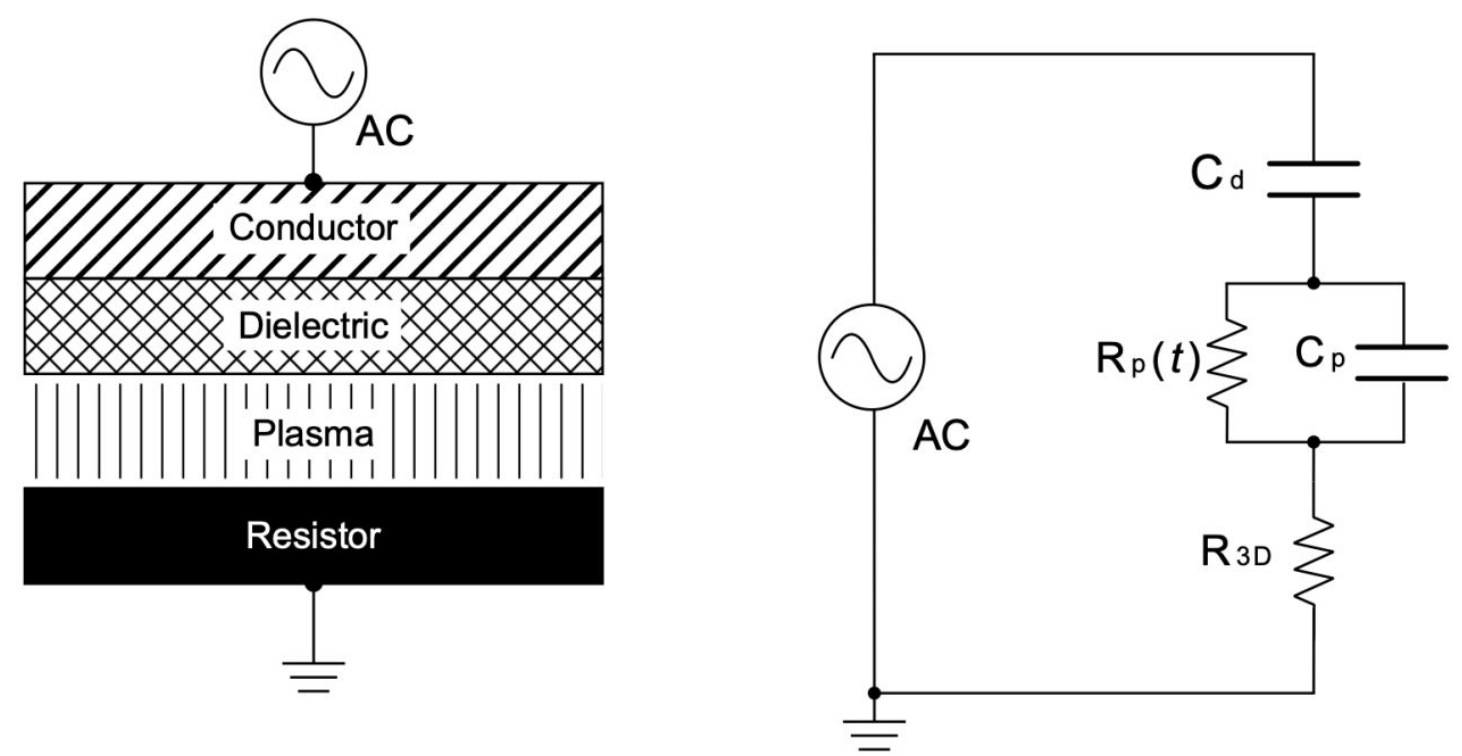

Figure S2: Electrical block diagram showing the flow of electric current from the AC source to the grounded printed part (left) and the corresponding circuit diagram depicting a basic electrical model of the elements critical to operation of the DBD applicator (right). 


\section{Section S4. Voltage and current waveform analysis}

Voltage and current traces were acquired using a LeCroy WaveRunner 204MXi oscilloscope with a $400 \mathrm{MHz}$ North Star PVM-4 high voltage probe and Bergoz current transformer for a bench-top (stationary) setup with a $3 \mathrm{~mm}$ air gap. The driving power supply was a custom AC power supply built in house. To determine the charge, the current was integrated numerically in Matlab and visually corrected for a linear offset. A parallelogram was fitted to the Lissajous (charge vs. voltage) data for capacitance and energy per pulse determination assuming the equivalent circuit from Figure S2. The rough parallelogram shape is characteristic of DBDs. The slight rounding of the corners comes from a more resistive discharge, in this case, due to the resistive load of the printed part. The slopes of the Lissajous figure correspond to the capacitance of the system. The lower slope $\left(\mathrm{C}_{\mathrm{tot}}\right)$ corresponds to the entire system capacitance which mostly consists of the air gap and the dielectric barrier in series; the upper slope $\left(\mathrm{C}_{\text {dielectric }}\right)$ corresponds to the system capacitance during a plasma discharge, where the main constituent is the dielectric barrier. 


\section{Section S5. Spectral analysis of plasma}

Broadband spectra was acquired with $100 \mathrm{~ms}$ integration time using a B\&W Tek BTC112E spectrometer that has a spectral resolution of $0.5 \mathrm{~nm}$. The sodium and potassium lines are assumed to be an impurity on the substrate or electrode. The narrow range spectra was acquired with an ICCD Stanford Computer Optics 4Picos detector attached to a $1 \mathrm{~m}$ focal length SPEX 1000M spectrometer. This system has a spectral resolution of $\sim 0.04 \mathrm{~nm}$. The $2^{\text {nd }}$ positive system of nitrogen was compared to modeled spectra from SpecAir to determine the rotational and vibrational temperatures of the nitrogen species in the gas. ${ }^{6}$ Non-equilibrium systems such as plasmas are characterized by multiple temperatures for the various energy modes of the system. For typical atmospheric plasmas the translational and rotational temperatures $\left(\mathrm{T}_{\text {trans }}\right.$ and $\left.\mathrm{T}_{\text {rot }}\right)$ are equal to each other and low compared to the vibrational temperature $\left(\mathrm{T}_{\text {vib }}\right)$. The electronic temperature $\left(\mathrm{T}_{\text {elec }}\right)$ is also typically higher than the vibrational temperature (thus $\mathrm{T}_{\text {trans }}=\mathrm{T}_{\text {rot }}<\mathrm{T}_{\text {vib }}$ $<\mathrm{T}_{\text {elec }}$ ). This type of spectroscopic temperature measurement is sensitive only to the light emitted from the discharge, thus the reported temperatures are only of the bright filaments of the discharge, not the average gas temperature, which is closer to ambient conditions. The filaments are also transitory, which also contributes to the disparity between the filament temperature and the average gas temperature. 


\section{Section S6. XPS Methodology}

Prior work: Narahara et al. reported the use of atmospheric pressure helium plasma plume for treating the layers of 3D-printed parts [16]. Although they noticed an increase in surface free energy of the PLA printed layers, it only resulted in modest improvements in interlayer strength.

Methods: XPS measurements were performed using an Omicron XPS system with Mg x-ray source. Control and freshly prepared plasma treated coupons were used for XPS analysis. High resolution XPS spectra of constituent elements was utilized to compute relative atomic composition using CasaXPS software version 2.3.16. Raw data are recorded in Figure S3.

Results: The carbon $(\mathrm{C} 1 \mathrm{~s})$, nitrogen $(\mathrm{N} 1 \mathrm{~s})$, and oxygen $(\mathrm{O} 1 \mathrm{~s})$ peaks are indicated on the XPS survey spectra in Figure S4A. Notably, the nitrogen peak for the plasma treated sample is more pronounced compared to the control sample. The peaks were resolved for percent atomic composition of the various functional groups on the surface (Figure S4B). It was found that before and after the plasma treatment the carbon peak decreased from $88.2 \%$ down to $81.6 \%$, the oxygen peak increased from $11.8 \%$ up to $12.7 \%$, and the nitrogen peak increased from null to $5.7 \%$. This indicates that the surface chemistry of the polyamide changes quite dramatically, with an increase in functional groups associated with high surface energy and favorable surface wettability of the surface for the next extruded polymer layer. Although the data suggests that conditions for polymer bonding will be more favorable in the presence of the plasma, it is unlikely that surface chemistry alone can account for the isotropic strength properties seen in our samples. A full polymer weld requires entanglement of the polymer chains across the weld interface. Thus, in addition to the initial wetting of the polymer interfaces, polymer mobility initiated by heating is required for a sufficient amount of time to reach full bond-line strength. 

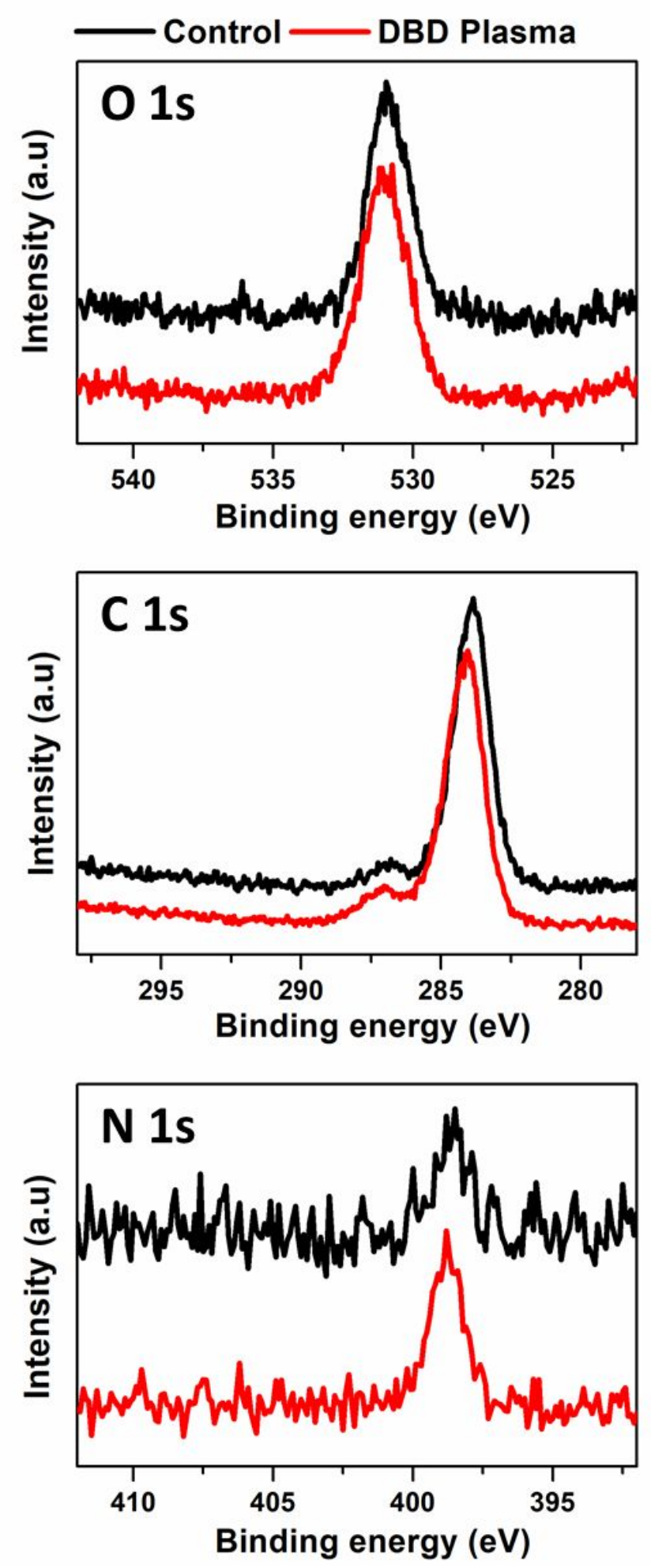

Figure S3: High resolution XPS spectra of oxygen, carbon, and nitrogen components of polymer surface before and after DBD plasma treatment. 
A.

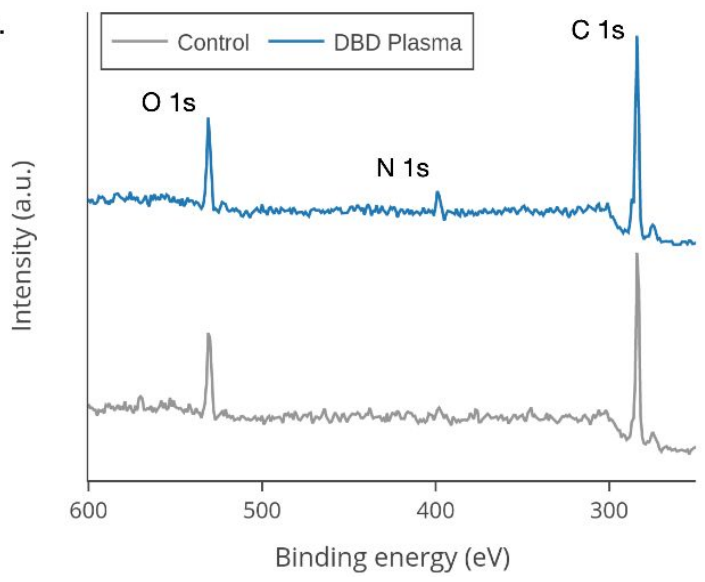

B.

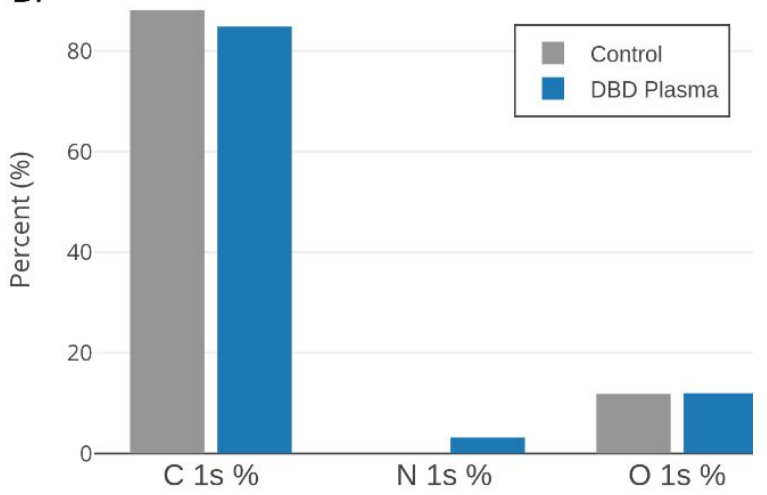

Figure S4. A. X-ray photoelectron (XPS) survey spectra of polymer surface before and after DBD plasma treatment. B. Change in the polymer surface atomic composition after DBD plasma treatment. 


\section{Section S7. Static fire test setup}

Static fire tests were used to determine the amount of heat energy contributed to the printed tensile plaque, by isolating the DBD plasma and electric field as the only energy input into the sample. This is in contrast to the normal operating mode of the DBD plasma on printed parts, where the residual heat of extruded plastic (and potentially the ambient temperature of a heated build chamber) contributes significantly to the observed thermal profile of the printed plaques. With this technique it is possible to establish precisely the effect of Joule heating on the sample and its contribution to the overall heat generated in normal tensile plaques. We printed plaques approximately $50 \mathrm{~mm}$ tall, $90 \mathrm{~mm}$ in width, and $3.2 \mathrm{~mm}$ in thickness then paused the print and cooled the print head in the middle of the sample. When the sample and build plate had cooled to room temperature, we supplied approximately $15 \mathrm{~W}$ of power to the DBD power supply to heat the sample for 20 seconds, then the power was shut off. The heating response was recorded with a FLIR camera (FLIR systems, A325SC) and analyzed in ResearchIR software (Figure S5, Movie S1).

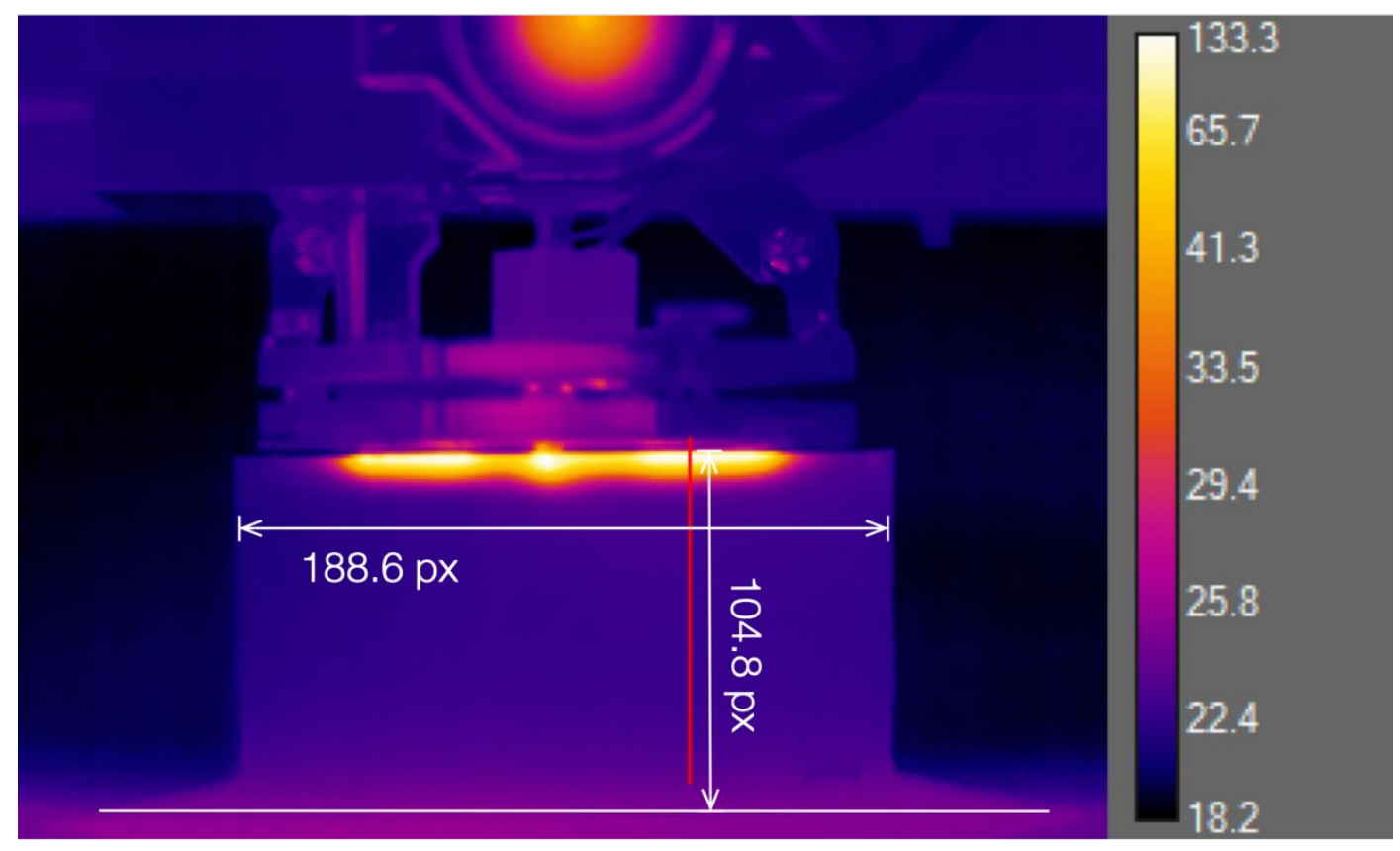

$$
\begin{aligned}
188.6 \mathrm{px} & =90 \mathrm{~mm} \\
104.8 \mathrm{px} & =50 \mathrm{~mm}
\end{aligned}
$$

Figure S5: FLIR image showing the temperature $\left({ }^{\circ} \mathrm{C}\right)$ distribution within the 3D-printed plaque (Side-view). The vertical red line corresponds to the temperature profile plotted in Figure 3A.

Movie S1: FLIR video showing the temperature $\left({ }^{\circ} \mathrm{C}\right)$ distribution within the 3D-printed plaque (Side-view) corresponding to Figure S5. 


\section{Section S8. Numerical analysis of static fire heating}

The simulation of static fire heating was carried out by solving the thermal energy balance which takes into account the volumetric heating and heat transfer in a 3D-printed plastic slab. The thermal energy balance is given by Equation 4 .

$$
\rho C_{p} \frac{\partial T}{\partial t}+\nabla \cdot \boldsymbol{q}+\frac{h}{d} \cdot\left(T-T_{e x t}\right)=Q \quad \ni \boldsymbol{q}=-k \nabla T,
$$

where $\rho$ is the mass density, $C_{p}$ is the heat capacity, $T$ is the temperature, $t$ is time, $\boldsymbol{q}$ is the heat flux within the sample, $h$ is the convective heat transfer coefficient out the sides of the sample, $\mathrm{d}$ is the thickness of a single layer, $Q$ is the volumetric heat source, and $k$ is the thermal conductivity.

The boundary condition is zero-flux at the bottom substrate; at the top surface and along the side, a convection boundary condition is applied as given by Equation 5, where $\mathbf{n}$ is the director normal to the boundary.

$$
\boldsymbol{- n} \cdot \boldsymbol{q}=h \cdot\left(T-T_{\text {ext }}\right),
$$

We neglect thermal gradients in the y-direction (the thin dimension of the slab). We simulate the time-dependent thermal profile of a given location in $\mathrm{x}$ as the DBD field passes over it with a specified residence time. The volumetric heat source decays exponentially as a function of distance (z) from the DBD disc.

$$
Q=Q_{0} \exp \left(-z / z_{0}\right)
$$

This lengthscale of $\mathrm{z}_{0}$ and the maximum heating rate $\mathrm{Q}_{0}$ are the only adjustable parameters.

Equation 4 is discretized in $\mathrm{z}$ using the finite difference method and an implicit multistep timestepper is used to solve for the temperature profile at each time step. This temperature profile is extruded forward in time to produce Figure 3B.

Table S1: The variables used for the numerical simulation are as follows:

\begin{tabular}{|c|c|c|}
\hline Variable & Value & Units \\
\hline $\mathrm{Q}_{0}$ & 7.9 & $\mathrm{~W} / \mathrm{m}^{3}$ \\
\hline $\begin{array}{c}\text { DBD residence } \\
\text { time }\end{array}$ & 20.3 & seconds \\
\hline $\mathrm{C}_{\mathrm{p}}$ & 1700 & $\mathrm{~J} /(\mathrm{kg} \mathrm{K})$ \\
\hline $\mathrm{k}$ & 0.25 & $\mathrm{~W} /(\mathrm{m} \mathrm{K})$ \\
\hline$\rho$ & 1150 & $\mathrm{~kg} / \mathrm{m}^{3}$ \\
\hline $\mathrm{T}_{\text {ext }}$ & 20 & ${ }^{\circ} \mathrm{C}$ \\
\hline $\mathrm{z}_{0}$ & 8 & $\mathrm{~mm}$ \\
\hline $\mathrm{h}$ & 5 & $\mathrm{~W} /\left(\mathrm{m}^{2} \mathrm{~K}\right)$ \\
\hline
\end{tabular}




\section{Section S9. Mechanical specimen printing and thermal analysis}

Rectangular plaques $90 \mathrm{~mm}$ in width, $135 \mathrm{~mm}$ long (z direction) and $3.2 \mathrm{~mm}$ thick were printed on a modified Stacker S4 3D printer, with a carbon nanotube composite polyamide filament (PA-Z, Essentium Inc.). The filament is composed of a multilayer structure where the outer surface is a polyamide nanotube composite and the core is neat polyamide. The GCode for the printed plaques (Figure S6) were generated using Simplify3D, and the header file is included below for reference. Print conditions for both the DBD and FFF control were identical, except for the DBD plasma applied to the DBD test samples. The PA filament was carefully dried for 16 hours at $100^{\circ} \mathrm{C}$, and then transferred to a custom dry box with molecular sieve desiccant and purged with dry nitrogen. While the samples were printed (Figure S7), the surface temperature of the plaques were recorded with a FLIR camera and a section of the heat profile $25 \mathrm{~mm}$ down from the surface was plotted.

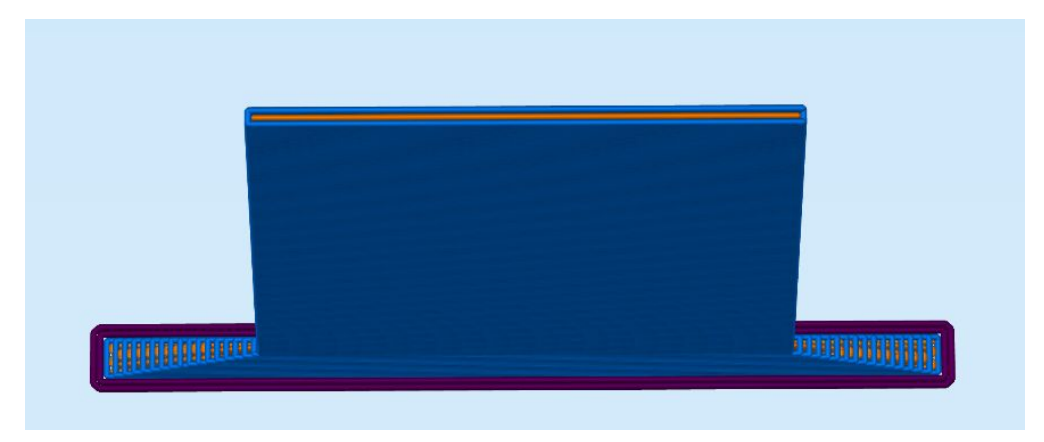

Figure S6: GCode visualization for printing pattern of ZX mechanical plaque 


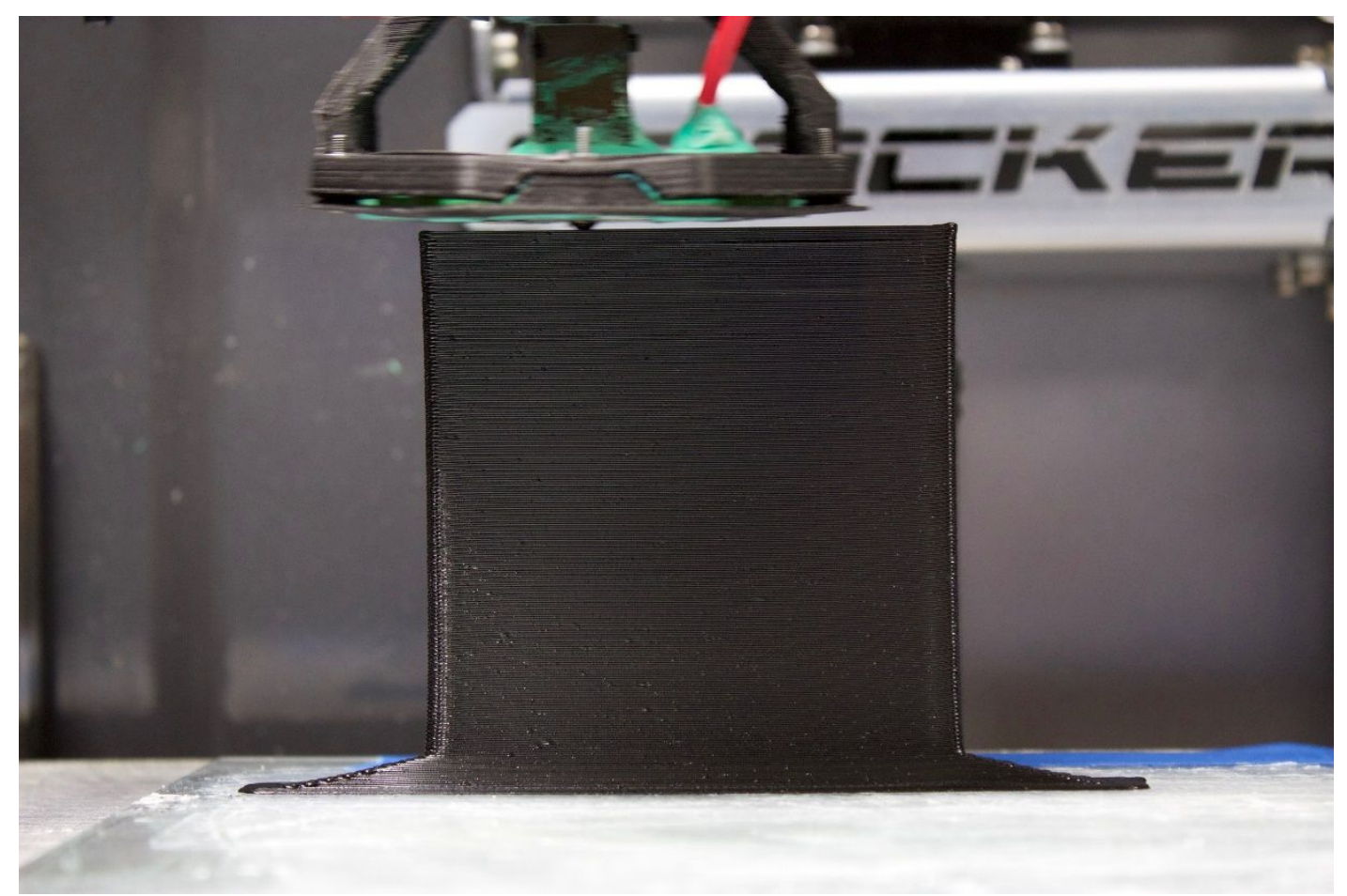

Figure S7: DBD-enhanced, 3D-printed plaque for mechanical testing. 


\section{$\underline{\text { GCode header }}$}

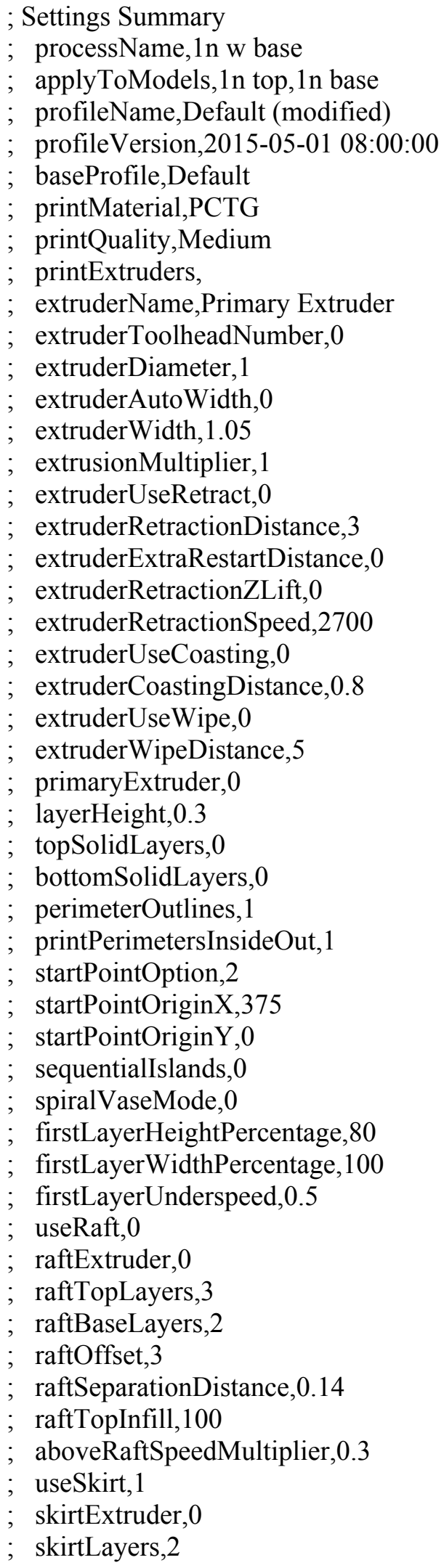




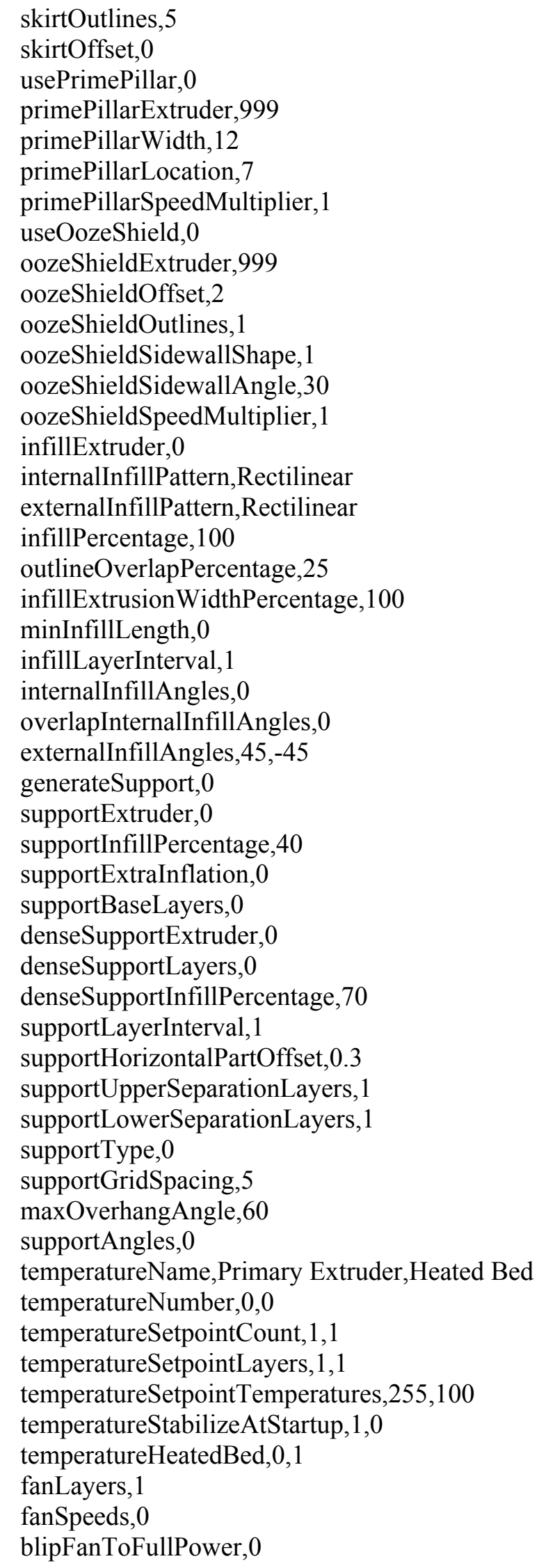




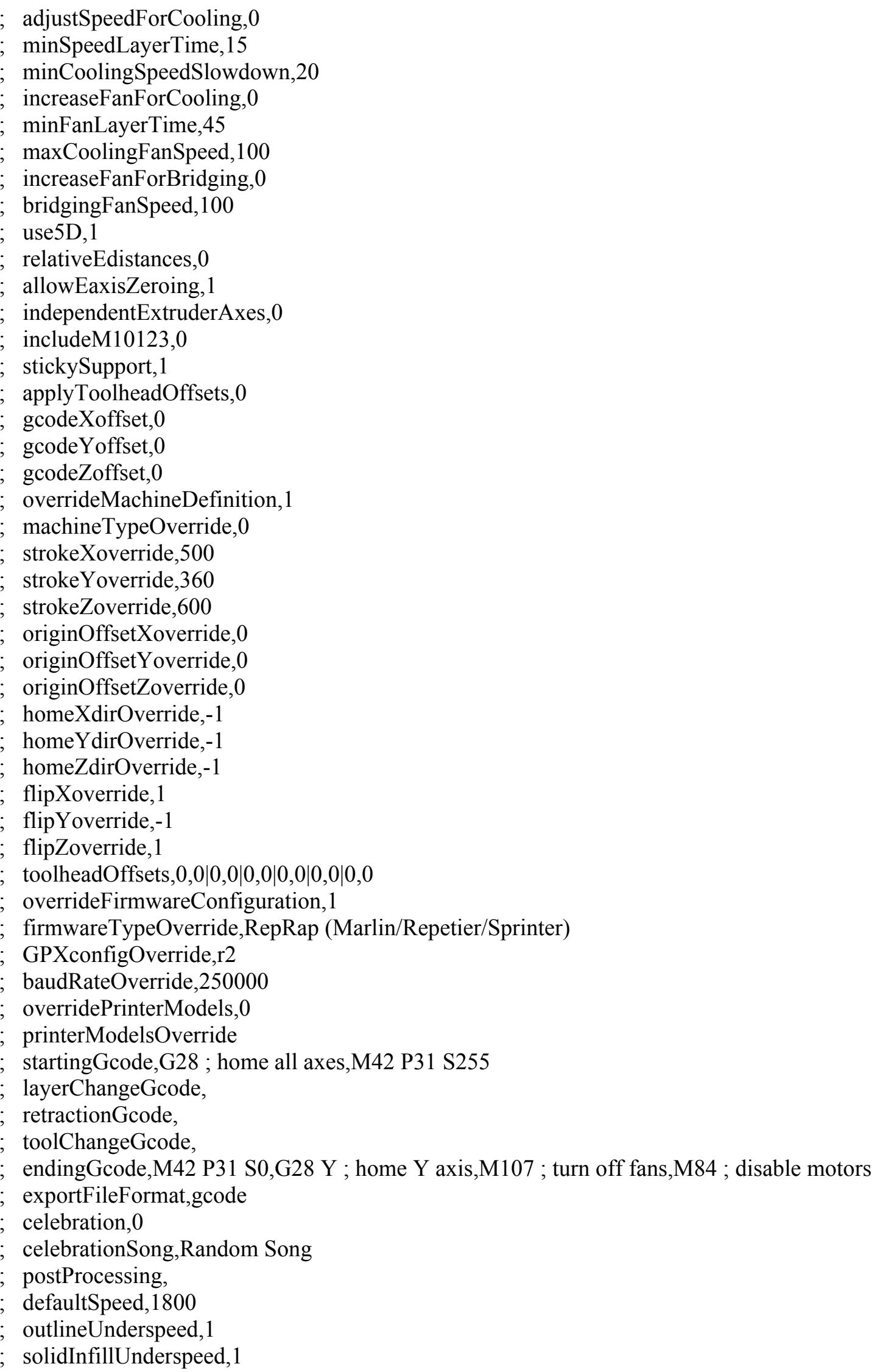




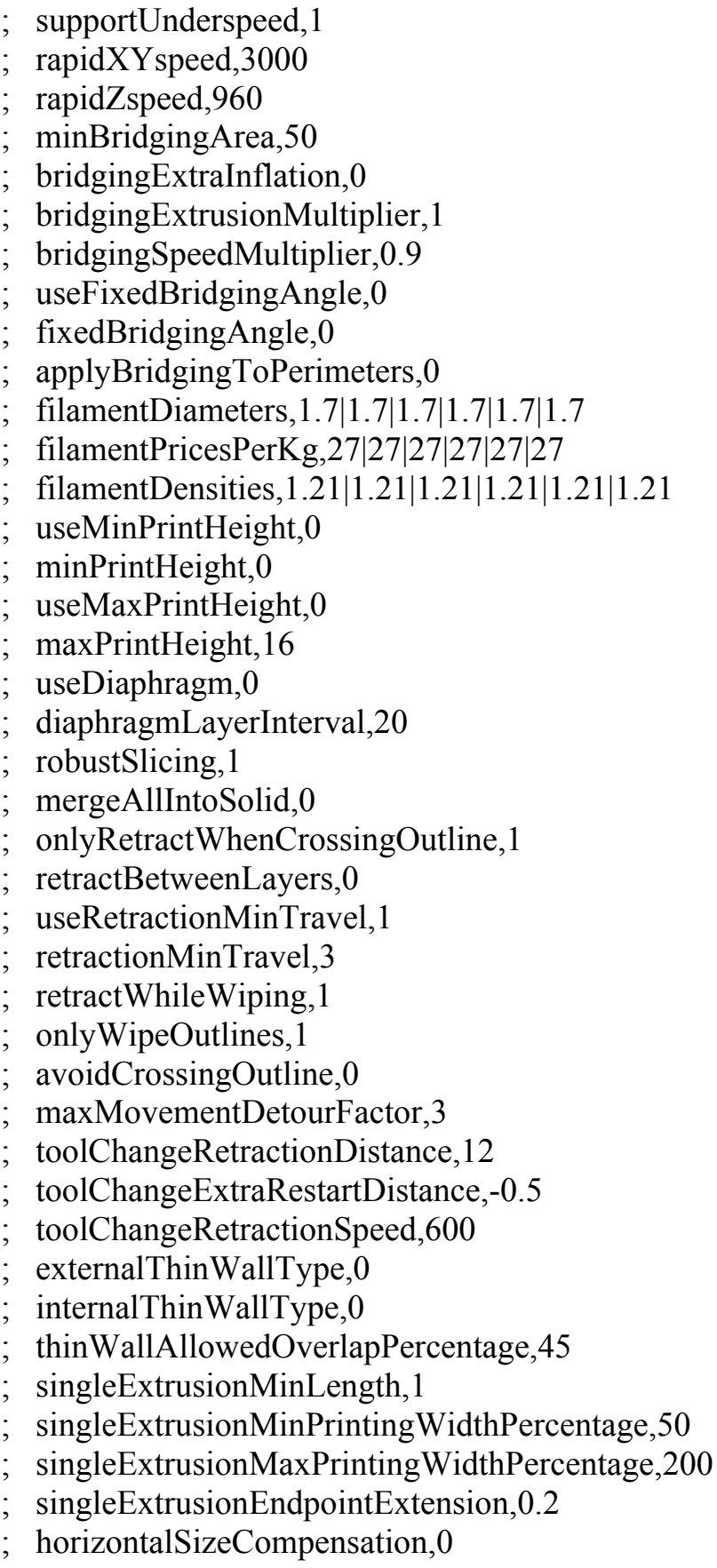




\section{Section S10. Injection molding and tensile testing}

Injection molded polyamide dogbones were prepared for comparison using an Arburg 320c Golden Edition injection molding machine. The corresponding 3D-printed dogbones were printed using the procedure above, followed by routing (Figures S8-S10). 


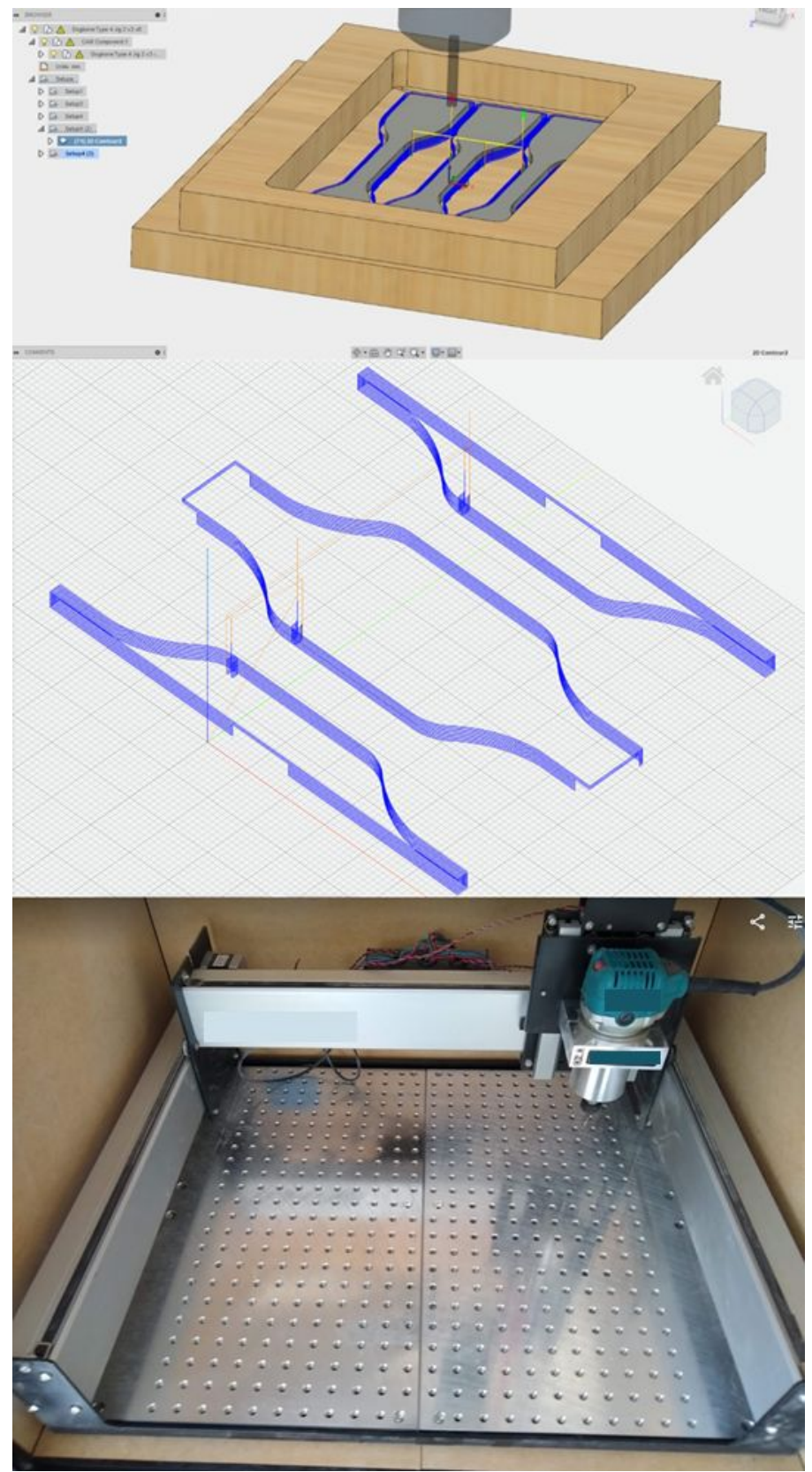

Figure S8: Completed 3D-printed plaques were CNC routed with a Shapeoko CNC router (Carbide 3D). Tensile samples were tested according to ASTM D638. 


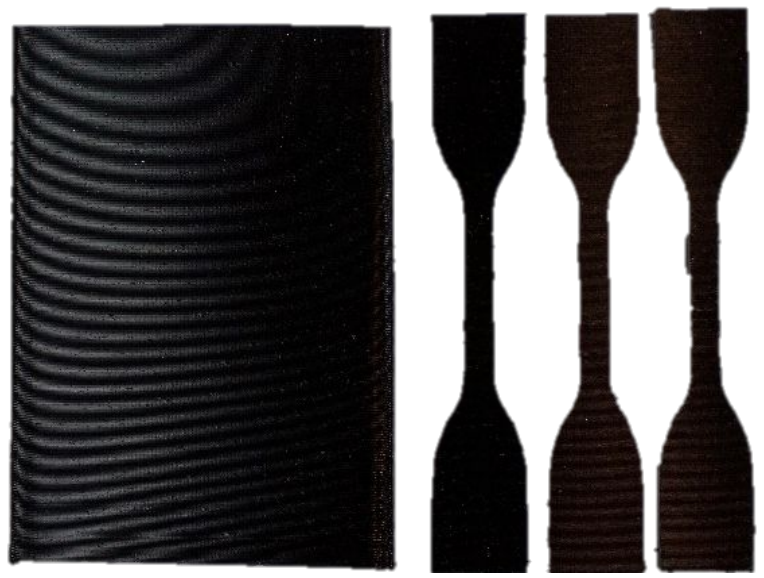

Figure S9: 3D-printed plaques before (left) and after (right) routing, according to ASTM D638. 

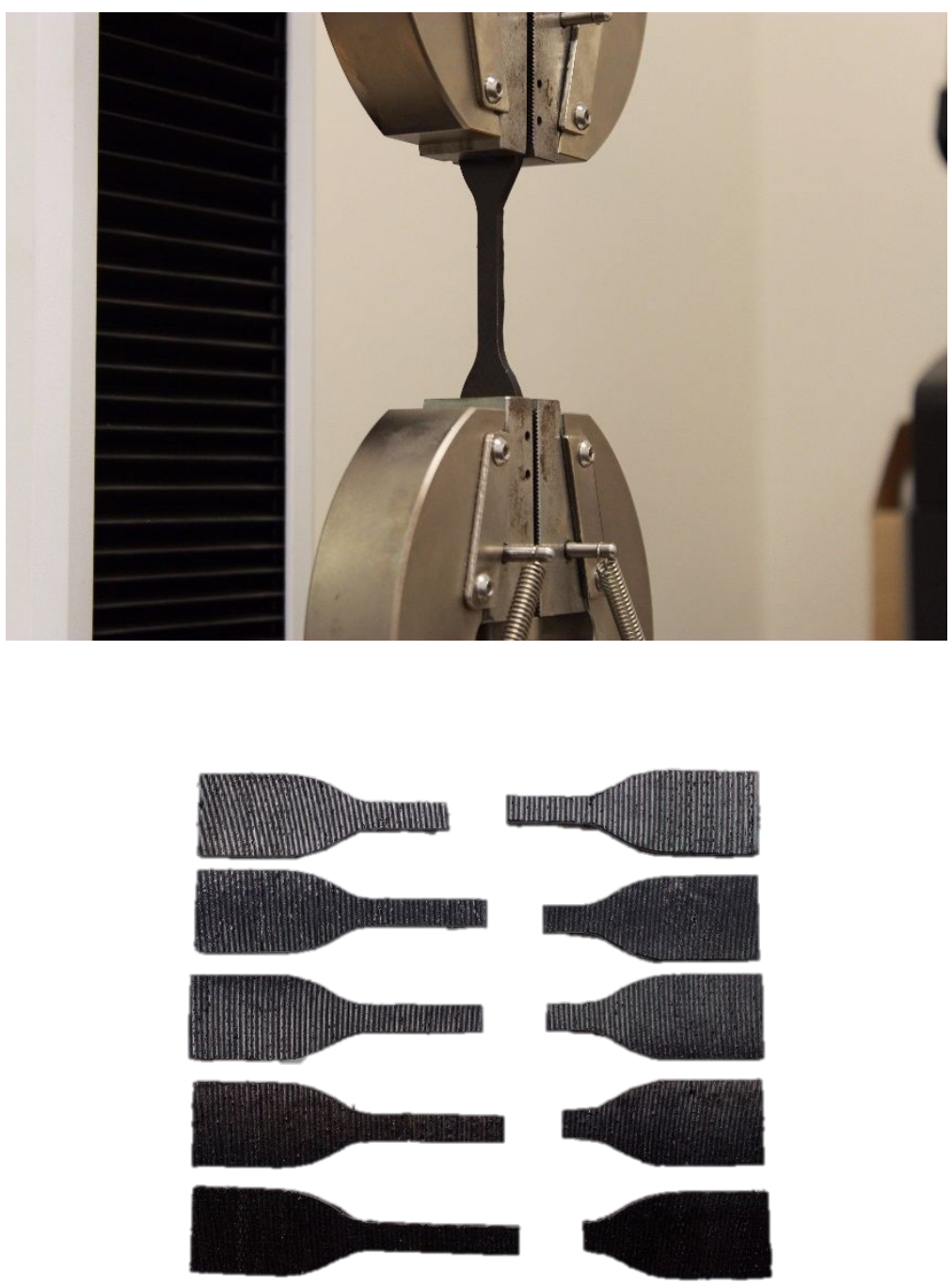

Figure S10: Mechanical dogbone samples were tested according to ASTM D638. 


\section{REFERENCES}

(1) Sweeney, C. B.; Moran, A. G.; Gruener, J. T.; Strasser, A. M.; Pospisil, M. J.; Saed, M. A.; Green, M. J. Radio Frequency Heating of Carbon Nanotube Composite Materials. ACS Applied Materials \& Interfaces 2018, 10, 27252-27259.

(2) Sweeney, C. B.; Lackey, B. A.; Pospisil, M. J.; Achee, T. C.; Moran, A. G.; Hicks, V. K.; Teipel, B. R.; Saed, M. A.; Green, M. J. Welding of 3D Printed Carbon Nanotube-Polymer Composites by Locally Induced Microwave Heating. Science Advances 2017, in press.

(3) Pipa, A.; Koskulics, J.; Brandenburg, R.; Hoder, T. J. R. o. S. I. The simplest equivalent circuit of a pulsed dielectric barrier discharge and the determination of the gas gap charge transfer. 2012, 83, 115112.

(4) Brandenburg, R. Dielectric barrier discharges: progress on plasma sources and on the understanding of regimes and single filaments. Plasma Sources Science \& Technology 2017, 26.

(5) Laroussi, M.; Alexeff, I.; Richardson, J. P.; Dyer, F. F. J. I. T. o. P. S. The resistive barrier discharge. 2002, 30, 158-159.

(6) Staack, D.; Farouk, B.; Gutsol, A. F.; Fridman, A. A. J. P. s. s.; technology. Spectroscopic studies and rotational and vibrational temperature measurements of atmospheric pressure normal glow plasma discharges in air. 2006, 15, 818. 